\title{
Correlation between MRI Findings and Arthroscopic Findings in Internal Degeneration of Knee
}

\author{
Mahibul Islam ${ }^{1}$, Rohit Ailani ${ }^{2}$, T.D.Bhattacharyya ${ }^{3}$, P.S.Chakrabarty ${ }^{4}$, \\ Sidhartha Baruah ${ }^{5}$ \\ 1,2,3,4,5 Dept Of Orthopaedics, Gauhati Medical College And Hospital,Guwahati
}

\begin{abstract}
Aim: The aim of this study is to compare the MRI findings with arthroscopy findings in internal derangement of knee.

Material and methods: 124 patients of different age group and sex with internal derangement of knee (IDK) were subjected to MRI for determining ACL, PCL, medial and lateral meniscus injury and later were subjected to arthroscopy. Accuracy of MRI diagnosis as had been made earlier was compared with arthroscopic examination findings.

Results: MRI performed on the injured knees revealed 90 tears of the menisci, of which 54 were of medial meniscus and 36 tears of lateral meniscus. There were 112 tears of the cruciate ligaments, out of which 104 were tears of ACL and 8 of PCL. Arthroscopy performed on the same knees revealed 78 tears of menisci, 48 of these were of medial meniscus and 30 of lateral meniscus. There were 118 tears of cruciates, of which 110 were of ACL and 8 of PCL. The accuracy of MRI in diagnosing acl injury is $89 \%$, for PCL is $100 \%$, for medial meniscus is $82.34 \%$ and for lateral meniscus is $78.16 \%$.

Conclusion: Magnetic resonance imaging is useful as a pre operative diagnostic tool in selected cases where a clinical examination cannot be performed as in acute injuries or in cases where clinical examination is inconclusive. The efficacy of MRI in diagnosing a tear varies among different intra articular structures.
\end{abstract}

\section{Introduction}

The knee joint is one of the most commonly injured joints, as an isolated injury or a frequent component in a multiple trauma patient. The knee is a complex joint, consisting of two condylar joints between the corresponding condyles of femur and tibia and a stellar joint between the patella and femur ${ }^{1}$.The principal intra-articular structures in knee are the two menisci, the two cruciate ligaments, and the two collateral ligaments. The menisci serve to distribute joint fluid, provides cartilage nutrition, mechanical shock absorption, increasing the surface area of the joint and therefore the stresses, serve to stabilize the joint, and as a weight bearing function. The cruciate ligaments function as stabilizers of the knee in both forward and backward motions of the tibia on the femur and provide an axis around which both medial and lateral rotatory movements are assisted ${ }^{2}$. The injury to these intraarticular structures is generally termed as "Internal derangement of knee" which was first coined by William Hey in $1784^{3}$.

Traumatic knee injuries were conventionally assessed clinically, and subjected to radiographs. In modern Orthopaedics, MRI and a subsequent arthroscopic procedure has come of age. A detailed clinical examination, with the numerous stability tests provide an almost $70 \%$ accuracy in diagnosing the pathology ${ }^{4,5,6}$. However, in the acute stage following injury, clinical tests may not be appropriate due to pain, thus an MRI is the preferred modality of investigation. It is non invasive, and considered to be highly sensitive to meniscal injuries ${ }^{7,8,9}$ but is less so for the Anterior cruciate ligament injuries. Arthroscopy of the knee is a key hole surgery performed as a daycare procedure. There are many indications however, this study is to correlate the findings subsequent to an MRI diagnosis.

\section{Aim And Objectives}

1. To determine the benefits of arthroscopy

2. To compare the sensitivity and specificity of MRI and Arthroscopy in diagnosing knee pathologies.

3. To find if MRI is mandatory in all IDKs.

\section{Material And Methods}

Total number of 124(116 males and 8 females)patients were selected who where clinically diagnosed to have an internal derangement of knee attending Gauhati Medical College and Hospital, Guwahati, India from May 2014 to November 2016, and who underwent an MRI, followed by Arthroscopy of the knee, are included in the study. A thorough history was taken and physical examination of the injured knee was performed. MRI 
examination were performed on all patients in the study, at our institute using a 1.5T MRI system. The scans were made in sagittal, coronal, and axial planes of the knee. All the sections were $3 \mathrm{~mm}$ thick. MRI images were reported on an objective proforma by a single senior consultant radiologist, who was blinded to the clinical findings. After reaching to a MRI diagnosis, the patients were subjected to an arthroscopy of the affected knee. The arthroscopic findings were recorded. Then the findings of MRI and arthroscopy were compared and analyzed. MRI diagnosis were placed into one of the four categories after arthroscopic evaluation ${ }^{11}: 1$. True positive: When MRI diagnosis of tear was confirmed on arthroscopic evaluation. 2. True negative: If the diagnosis of no tear was confirmed on arthroscopy. 3. False positive: If MRI showed a tear but arthroscopy was negative 4. False negative: If MRI images were negative but arthroscopy showed a tear. Based on the above categories, four parameters were calculated to assess the reliability of the MRI results ${ }^{12}$ : 1 . Sensitivity: sensitivity of MRI is the ability of the MRI to detect an abnormality. It is determined by the equation : Truepositive/ (true-positive+true negative) X 100 per cent. 2. Specificity: specificity of MRI is the ability of MRI to give how many detected tears are usually accurate. It is determined by the equation: True-negative/ (true negative+false positive) $X 100$ per cent. 3. Positive predictive value: It correlates a positive result of MRI with findings of arthroscopy. It is calculated by the equation: True-positive/ ( true-positive+false positive) X 100 per cent. 4. Negative predictive value: It correlates a negative result on MRI with the findings of arthroscopy. It is calculated by the equation: True-negative/ ( true-negative+false-negative) X 100 per cent.

\section{Results}

The results obtained from MRI were compared to those of arthroscopic findings. Magnetic resonance imaging performed on the injured knees revealed 90 tears of the menisci, of which 54 were of medial meniscus and 36 tears of lateral meniscus.. There were 112 tears of the cruciate ligaments, out of which 104 were tears of ACL and 8 of PCL. Arthroscopy revealed 78 tears of menisci, 48 of these were of medial meniscus and 30 of lateral meniscus. There were 118 tears of cruciates, of which 110 were of ACL and 8 of PCL.

\begin{tabular}{|l|l|l|l|l|}
\hline Test & True positive & True negative & False positive & False negative \\
\hline ACL MRI findings & 101 & 12 & 2 & 8 \\
\hline PCL MRI findings & 8 & 116 & 0 & 0 \\
\hline Medial meniscus MRI findings & 40 & 62 & 14 & 8 \\
\hline Lateral meniscus MRI findings & 22 & 78 & 16 & 8 \\
\hline
\end{tabular}

With regards to tears of medial meniscus, comparison of the findings of magnetic resonance imaging with those of arthroscopy revealed 40 true positive, 62 true negative, 14 false positive and 8 false negative images. Sensitivity was 83.33 per cent, specificity was 81.58 per cent, positive and negative predictive values were 81.90 and 83.03 per cent respectively.For the lateral meniscus, there were 22 true positive, 78 true negative, 16 false positive and 8 false negative results. Sensitivity was 73.33 , specificity was 82.99 per cent. Positive and negative predictive values were 81.16 and 75.66 per cent respectively.For the anterior cruciate ligament, there were 101 true positive, 12 true negative, 2 false positive and 8 false negative results. Sensitivity was 92.7 percent, specificity was 85.71 percent and positive and negative predictive values were 86.65 and 92.18 percent respectively. For the posterior cruciate ligament, there were 8 true positive and 116 true negative results. There were no false positive, and false negative results and sensitivity is $100 \%$, specificity is $100 \%$, positive predictive value $100 \%$ and negative predictive value were $100 \%$.

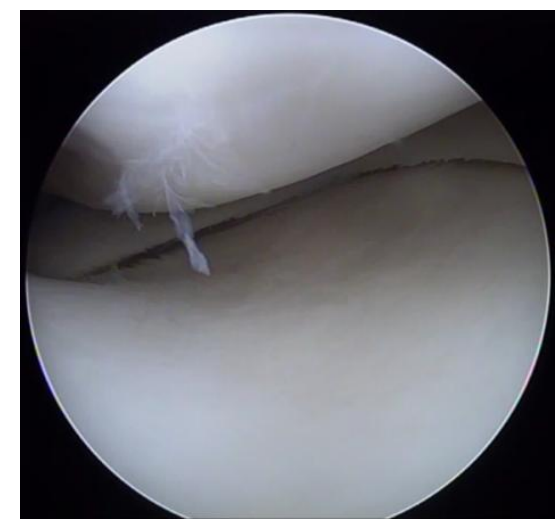

Arthroscopy Showing

Intact Medial Meniscus

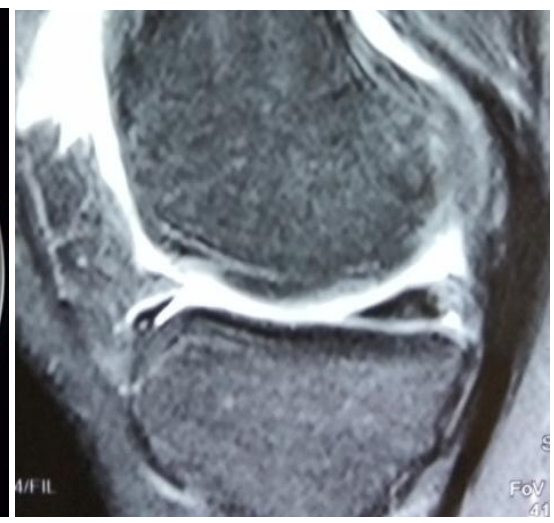

Mri Showing

Torn Medial Meniscus 


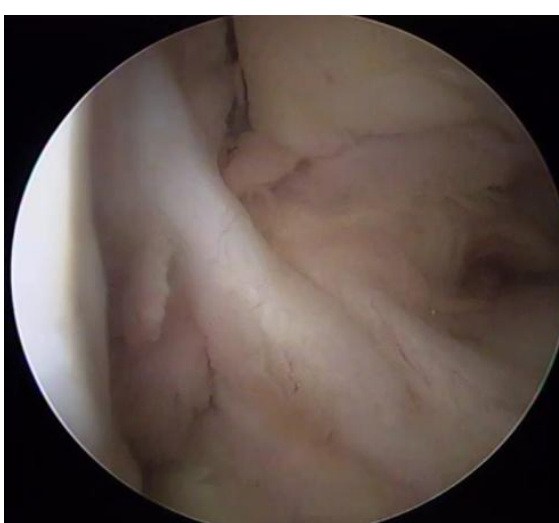

Arthroscopy Showing

Acl Tear

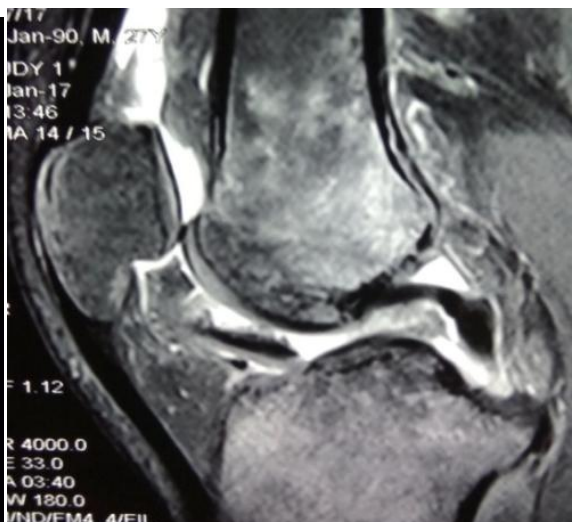

Mri Showing

Acl Tear

\section{Discussion}

Imaging of the menisci showed 30 false positive and 16 false negative results of which 14 false positive results were of medial meniscus, and 16 of lateral meniscus, 8 false negative images were of medial meniscus and 8 of the lateral meniscus. Among the false positive results of meniscus, many showed significant fraying due to degeneration, which was reported as a tear on MRI. Imaging of the cruciate ligaments in this study showed 2 false positive and 8 false negative results, all of which were for the anterior cruciate ligament. There were no false positive or false negative results for the PCL in this study. The false positive results for ACL were attributed to the presence of large ligamentum mucosum, which was reported as a tear in the substance of ACL. In 5 of the 8 false negative results, the ACL was found to be lax on probing, which was probably due to a partial tear, and 3 tear was proximal and ACL was found attached to the PCL, which was hence reported as normal.

The results of this study is in accordance to the literature which suggests an accuracy of 68 to 88 percent for the meniscal tears ${ }^{14}$ and 80 to 94 percent for the cruciate ligament tears ${ }^{15}$. Various intra articular pathologies of the knee such as loose bodies, chondral fractures, degenerative changes, plicae can mimic a meniscal tear. Mucoid and eosinophilic degeneration can cause false positive results on MRI ${ }^{14}$. Post operative cases with metallic implants around the knee produce artifacts on MR images and an erroneous interpretation of the images is likely in such cases. Magnetic resonance imaging is useful as a diagnostic tool in internal derangements of the knee. It is useful in circumstances where there is a need for detailed differential diagnosis. It is also an important diagnostic tool in cases of acute and painful knees, where clinical examination is difficult to perform. Currently MRI is gaining popularity as a diagnostic tool in knee injuries due to increasing sports injuries, and road traffic accidents.

A further improvement in the techniques and increasing experience in interpretation of the images is likely to reduce the false positive and false negative results in future. Magnetic resonance imaging also helps the surgeon to plan the definitive management of a tear during the same session.

\section{Conclusion}

Knee joint injuries are common.The need to accurately evaluate the knee injuries is very crucial for the proper management and outcome; otherwise it will lead to chronic disability to the patient.MRI is useful as a pre operative diagnostic tool in selected cases where a clinical examination cannot be performed as in acute injuries or in cases where clinical examination is inconclusive.The efficacy of MRI in diagnosing a tear varies among different intra articular structures. MRI has a high accuracy in diagnosing a tear of PCL.Sensitivity for medial meniscal tear is higher as compared to lateral meniscus and high for PCL as compared to ACL.MRI has a high positive predictive value for ACL, but has a low negative predictive value.For PCL tears, MRI has a high negative predictive value which indicates that with a negative result for PCL on MRI, a diagnostic arthroscopy can be avoided. However it is to be noted that reporting of MRI is dependent on the technician and the Radiologist and has a long learning curve. From this study, we believe that routine interaction of arthroscopist and radiologist with retrograde introspection about the diagnosis should be encouraged in the everyday practice,based on clinical examination that comes first, surgeons decide whether he must proceed to further laboratory tests, MRI, conservative or surgical treatment.

\section{References}

[1]. Peter L Williams, Roger Warwick : Arthrology; Gray’s anatomy 36th ed. 482

[2]. Robert H, Miller III, Frederick M.Azar :Knee injuries; Campbell's operative orthopaedics. 11th ed. 2410.

[3]. Hughston JC: Acute knee injuries in athletes, Clin Orthop 23:114,2962. 
[4]. Terry GC, Tagert BE, Ypung MJ. Reliability of the clinical assessment in predicting the cause of internal derangement of the knee. Arthroscopy 1995;11:568-576

[5]. Abdon P, Arnbjornsson AH, Egrund N, Lindstrand A, Odenbring S, Pettersson H. Lateral meniscal lesions in patients with clinically suspected medial lesions, Acta Orthop Scan 1989;60:453-456.

[6]. Munk B, Madsen F, Lundorf E: Clinical MRI and arthroscopic findings in knees. Journal of Arthroscopy. 1998 March;4(2):171-5.

[7]. Gillies, Hamish, Seligson, Dravid: Precision in diagnosis of meniscal lesions: A comparision of clinical evaluation, Arthrography, and arthroscopy. J. Bone and joint surg., 61-A: 343-346, April 1979

[8]. Edwin H. G. Oei, Msc, Jeroen J. Nikken, MD, Antonia C. M. Verstijnen, Msc, Abida Z. Ginai, MD, PhD and M.G. Myriam Hunink, MD, PhD: MR imaging of the menisci and cruciate Ligaments: A systematic review. Radiology 2003;226:837-848.

[9]. Quinn SF, Brown TF. Meniscal tears disgnosed with MR imaging versus arthroscopy: how reliable a standard is arthroscopy? Radiology 1991; 181:843-847.

[10]. Barry KP, Mesgarzadeh M, Triolo J, Moyer R, Tehranzadeh J, Bonakdarpour A. Accuracy of MRI patterns in evaluating anterior cruciate ligament tears. Skeletal Radiol 1996;25;365-370.

[11]. Fleiss JL. The statistical basis of meta-analysis. Stat Methods Med Res. 1993;2(2):121-45.

[12]. Mackenzie R, Palmer CR, Lumas DJ. Magnetic resonance imaging of the knee: diagnostic performance studies. Clin Radiol 1996; 51:251-255.

[13]. Moses LE, Shapiro D, Littenberg B.Combining independent studies of a diagnostic test into a summary ROC curve: data-analytic approaches and some additional considerations. Stat Med. 1993 Jul 30;12(14):1293-316.

[14]. Herman LJ, Beltran J: Pitfalls in MR imaging of the knee, Radiology 167:775,1988.

[15]. Tung GA, Davis LM, Wiggins ME, Fadale PD.Tears of the anterior cruciate ligament:Primary and secondary signs at MR imaging.Radiology,1993;188:661-7. 